Ann. Abeille, I962, 5 (I), 65-67.

\title{
EXAMEN HISTOLOGIQUE DES VIEUX RAYONS A PAROIS NOIRES ET ÉPAISSIES
}

\author{
Madeleine FRICHOT-RIERA. \\ Station de Recherches sur l'Abeille et les Insectes sociaux, \\ Bures-sur-Yvette (Seine-el-Oise)
}

SOMMAIRE

Les vieux rayons se coupent facilement au microtome ; ils sont composés de cire qui repose sur une trame donnant les réactions histochimiques des pectines et des matières protéiques, des phos. phates et aussi du glycogène; mais la coloration de Best se produit dans ce cas après la digestion salivaire.

Les rayons des Abeilles, lorsqu'ils ont séjourné pendant plusieurs années dans la ruche, deviennent noirs, lourds et leurs parois s'épaississent beaucoup. Chauvin a montré que la matrice de cire initiale s'encrasse de toute sortes de composés, les uns hydrocarbonés, et les autres azotés. Mais la chimie ne saurait nous indiquer la répartition de tous ces corps sur la cire, et c'est pourquoi nous avons entrepris l'étude histologique des vieux rayons.

\section{MÉTHODE}

Après toutes sortes d'essais d'enrobage infructueux, nous nous sommes aperçus que pour obtenir des coupes convenables au microtome à paraffine, il suffisait tout simplement de coller le fragment de rayon sur le porte-objet. Les bords des cellules se coupent exactement comme un bloc de paraffine, et on peut facilement les monter sur lames. Nous avons choisi le bord des cellules et n'avons fait que peu de coupes passant par le fond; en effet la masse des cocons et les excréments des larves durcis entre leurs parois rendent la coupe très difficile ou impossible. De la même façon et sans aucune précaution ni inclusion préalable il est possible d'obtenir de bonnes coupes par congélation au microtome de Sartorius.

\section{RÉSULTATS}

Lorsque les coupes sont examinées telles quelles tout de suite après la coupe, il n'y a pas grand'chose à en dire. Elles sont de couleur foncée, même sous une faible épaisseur, et fortement biréfringentes; en montant les coupes dans la glycérine, on s'aperçoit comme il fallait s'y attendre, que la cire n'est pas homogène : on y discerne 
de petites Ianguettes rapprochées irrégulièrement les unes des autres et qui correspondent sans doute aux plaquettes de sécrétion cirière que les Abeilles collent les unes aux autres. Au milieu de la cire, beaucoup d'impuretés viennent s'insérer : grains de pollen entiers, très nombreux, et débris plus nombreux encore, qui se colorent pour la plupart au vert d'iode et paraissent de nature végétale; ils prennent aussi le carmin aluné.

Les coupes de rayons ont été collées à l'eau albumineuse, suivant la technique habituelle; il est donc facile d'enlever la cire au moyen du xylène et du toluène. La coupe privée de cire et montée au baume permet de découvrir, avec les mêmes grains de pollens et débris variés un trait de structure inattendu : la présence d'une sorte de trame formée de corps oblongs, irréguliers aux limites plus ou moins distinctes; on dirait, pour employer une image audacieuse, le squelette des plaquettes de cire que nous décrivions tout à l'heure.

Cette trame est grisâtre, noirâtre sous forte épaisseur ; elle parait contenir les pigments foncés des vieux rayons; elle est plus ou moins soluble dans l'eau, disparait dans l'eau alcaline, paraît insoluble dans l'alcool. Nous avons tenté sur cette trame diverses colorations histochimiques.

La réaction de Salazar, au tannin et au chlorure ferrique, qui colore en noir les matières protéiques, a donné des résultats positifs et très intenses sur la trame. Le rouge de ruthénium, qui colore intensément la trame, caractérise d'habitude les matières pectiques ; mais par ailleurs, ChaUvin signale que, d'après ULRICH, on ne peut caractériser de pectines dans les eaux d'extraction des vieux rayons ; nous ne savons donc comment interpréter la réaction histochimique. La recherche du glycogène, à l'aide de la réaction au tannin-safranine, a été positive également, toujours sur la trame; mais nous avons voulu la confirmer au carmin de Best, sur témoin soumis à l'action des diastases salivaires ; malheureusement le Best est positif ici après action de la salive; ainsi donc on ne peut affirmer que la substance de la trame contienne du glycogène.

Nous avons recherché sans résultat les ozasones et peroxydases, les acides aminés et peptides par la ninhydrine et l'alloxane. Après microincinération d'une coupe à $700^{\circ}$, la cire s'étant volatilisée ainsi que les composés organiques, il reste une trame blanche collée à la lame ; l'analyse minérale, dont les résultats figurent dans le travail de Chauvin, montre l'abondance du calcium et du phosphore. Nous avons donc recherché les phosphates par le réactif de Lilienfeld et Monti (à 1'acide molybdique, ammoniaque, acide nitrique) dans la version de Win'tER et SMíTH (I922). Après le passage final au ferricyanure de potassium, nous voyons apparaitre une multitude de cristaux cubiques bleu-verts ; nous en avons conclu à la présence de phosphates.

\section{DISCUSSION}

Il nous semble que le résultat le plus intéressant est la présence d'une trame sousjacente à la cire et d'une matière bien différente. D'après les quelques essais réalisés sur des rayons assez récents (mais les coupes sont bien plus difficiles que dans le cas des vieux rayons) il semble que cette trame existe aussi chez eux. A notre avis elle doit provenir au moins en partie d'une sécrétion des glandes salivaires des ouvrières lorsqu'elles triturent la cire fraîchement sécrétée pour 1'incorporer au rayon en construction. Il est vrai qu'on sait peu de chose à ce sujet; car si l'anatomie et quelques 
points de la physiologie de la glande labiale (qui est utilisée, croit-on, dans le travail de la cire) sont bien connus (Simpson, I960) il n'en est pas de même du travail des lamelles de cire avec ou sans l'aide de cette sécrétion.

Les matériaux complexes signalés par CHaUvin dans les vieux rayons sont-ils tous incorporés à la trame? Il est difficile de l'affirmer ; d'après nos coupes, ils semblent toutefois qu'il ne se trouve rien de particulier à la surface le plus externe des parois de cire; leur contexture est homogène de la périphérie au centre. Comme certaines réactions de solubilité de la trame correspondent assez bien à celles signalées par Chauvin dans son extrait hydrosoluble de vieux rayons, nous pencherions finalement vers l'hypothèse que ce matériel réside dans la trame.

Une question non résolue est celle de la pectine ; certains caractères de l'extrait sec évoqués par Chauvin (réactions de solubilité, de précipitation, tendance de l'extrait mou à s'étirer en longs fils) auraient pu à la rigueur s'interpréter dans le cas d'une pectine ; mais UrRICH n'a pu la caractériser ; et pourtant nous obtenons une réaction positive au rouge de ruthénium (nous convenons que sa valeur n'est pas absolue).

Reģu pour publication en juin 1961.

\section{SUMMARY}

The old combs are easily cut with a microtome; thry consist of wax deposited on a weft and give histochemical reactions of pectin and proteins, phosphate and glycogen (but in this case the Best'colour is seen also after salivary digestion).

\section{RÉFÉRENCES BIBLIOGRAPHIQUES}

Sumpson J., ig6o. The functions of the salivary glands of Apis mellifera. J. Insed. Physiol., 4, ıо7-I2 r. 